\title{
Management Strategies and Outcomes for Hyponatremia in Cirrhosis in the Hyponatremia Registry
}

\author{
Samuel H. Sigal (D), ${ }^{1}$ Alpesh Amin, ${ }^{2}$ Joseph A. Chiodo III, ${ }^{3}$ and Arun Sanyal ${ }^{4}$ \\ ${ }^{1}$ Department of Medicine, Montefiore Medical Center and Albert Einstein College of Medicine, Bronx, New York 10467, USA \\ ${ }^{2}$ Department of Medicine, University of California, Irvine, California 92868, USA \\ ${ }^{3}$ Agile Therapeutics, Inc., Princeton, New Jersey 08540, USA \\ ${ }^{4}$ Virginia Commonwealth University Medical Center, Richmond, Virginia 23298, USA \\ Correspondence should be addressed to Samuel H. Sigal; ssigal@montefiore.org
}

Received 2 March 2018; Revised 31 July 2018; Accepted 8 August 2018; Published 27 September 2018

Academic Editor: En-Qiang Chen

Copyright (c) 2018 Samuel H. Sigal et al. This is an open access article distributed under the Creative Commons Attribution License, which permits unrestricted use, distribution, and reproduction in any medium, provided the original work is properly cited.

\begin{abstract}
Aim. Treatment practices and effectiveness in cirrhotic patients with hyponatremia (HN) in the HN Registry were assessed. Methods. Characteristics, treatments, and outcomes were compared between patients with $\mathrm{HN}$ at admission and during hospitalization. For $\mathrm{HN}$ at admission, serum sodium concentration [Na] response was analyzed until correction to $>130 \mathrm{mmol} / \mathrm{L}$, switch to secondary therapy, or discharge or death with sodium $\leq 130 \mathrm{mmol} / \mathrm{L}$. Results. Patients with $\mathrm{HN}$ at admission had a lower [Na] and shorter length of stay (LOS) than those who developed $\mathrm{HN}(P<0.001)$. Most common initial treatments were isotonic saline (NS, 36\%), fluid restriction (FR, 33\%), and no specific therapy (NST, 20\%). Baseline [Na] was higher in patients treated with NST, FR, or NS versus hypertonic saline (HS) and tolvaptan (Tol) $(P<0.05)$. Treatment success occurred in $39 \%, 39 \%, 52 \%, 78 \%$, and $81 \%$ of patients with NST, FR, NS, HS, and Tol, respectively. Relapse occurred in $55 \%$ after correction and was associated with increased LOS (9 versus 6 days, $P<0.001) .34 \%$ admitted with $\mathrm{HN}$ were discharged with $\mathrm{HN}$ corrected. Conclusions. Treatment approaches for HN were variable and frequently ineffective. Success was greatest with HS and Tol. Relapse of HN is associated with increased LOS.
\end{abstract}

\section{Introduction}

Dilutional hyponatremia (HN) is a frequent consequence of severe portal hypertension in cirrhosis. It is the result of severe vasodilation, leading to increased arginine vasopressin (AVP) release and consequent water retention $[1,2] . \mathrm{HN}$ is especially common in the hospitalized patient [3] and is associated with severe ascites, hepatic encephalopathy (HE), and impaired renal function. In a retrospective study of 20,000 patients, HN was predictive of worsening disease, mortality, a higher 30-day readmission rate [4], and a 1.74day increase in average hospital length of stay (LOS).

Management options for $\mathrm{HN}$ include discontinuation of diuretics, fluid restriction (FR), and administration of isotonic saline (NS), hypertonic saline (HS), or a vasopressinreceptor antagonist (or "vaptan"). FR is usually the first treatment used but is limited by patient adherence. Administration of NS and HS is problematic as they exacerbate fluid overload and ascites. Vaptans block the actions of AVP at vasopressin-2 receptors in cells of the renal collecting duct and provide a targeted approach to treatment in patients with inappropriately elevated AVP levels $[5,6]$. There are currently two FDA-approved vaptans: conivaptan (Cumberland Pharmaceuticals, Inc., Nashville, Tennessee, USA) is a dual vasopressin-1A/2-receptor antagonist available for IV use, and tolvaptan (TO; Otsuka Pharmaceutical Co., Ltd., Tokyo, Japan) is an oral selective vasopressin-2-receptor antagonist $[5,6]$. Both are indicated for euvolemic and hypervolemic HN. Cirrhosis was initially an approved indication for TO but was subsequently removed due to the development of hepatocellular injury during an investigational study of its use in autosomal dominant polycystic kidney disease [7].

The effectiveness of treatment strategies and impact on LOS for hospitalized cirrhotic patients with HN have not been previously reported. The HN Registry (NCT01240668) is an observational, multicenter, real-world study of patients 
hospitalized with euvolemic or hypervolemic HN. The objectives were to obtain clinical characteristics of patients and assess treatment practices, effectiveness, and resource utilization using LOS as a surrogate. The results of the entire population have previously been published [8]. In that report, the management and response of all patients with $\mathrm{HN}$ regardless of underlying condition were reported with only mention of the percentage of patients with cirrhosis. This analysis specifically assessed the subpopulation of patients with cirrhosis.

\section{Materials and Methods}

2.1. Study Design. Data from cirrhotic patients enrolled in the HN Registry [8] without concomitant nephrotic proteinuria, severe cardiomyopathy (ejection fraction $<50 \%$ ), or severe azotemia (creatinine $\geq 3.0 \mathrm{mg} / \mathrm{dL}$ ) were entered in a database that included clinical characteristics, laboratory results, volume of fluid intake and output over each 24-hour period (if available), amount of FR, treatment with IV NS and HS, diuretics, medications used to treat $\mathrm{HN}$, paracentesis, and LOS [8]. Severity of ascites and hepatic encephalopathy (HE) were recorded, and Child-Pugh scores, MELD, and MELD-Na scores were calculated [9]. Patients were classified as having $\mathrm{HN}$ diagnosed at the time of hospital admission versus hospital-acquired $\mathrm{HN}$ and categorized as mild $\left(\left[\mathrm{Na}^{+}\right]\right.$ $>125-130 \mathrm{mmol} / \mathrm{L})$, moderate $(120-125 \mathrm{mmol} / \mathrm{L})$, and severe $(<120 \mathrm{mmol} / \mathrm{L})$.

Initial treatment for $\mathrm{HN}$ was recorded. FR was based on an order by the treating physician. NS treatment was defined as administration of $>500 \mathrm{ml} \mathrm{NS}$ over a 24 -hour period. No specified therapy (NST) was defined as observation for $\geq 2$ days without a specific treatment. Patients who received NS, $\mathrm{HS}$, or TO alone or in conjunction with FR were combined. A 1-day gap of no therapy between 2 treatment episodes constituted the end of initial treatment except for patients receiving TO in which case a 1-day gap was permitted. The study was exclusively observational, and treatment was solely determined by the treating physician.

Response to therapy for patients admitted with HN was assessed daily. On each day, patients were categorized based on $\mathrm{HN}$ severity and achievement of a treatment endpoint (correction to $\left[\mathrm{Na}^{+}\right]>130 \mathrm{mmol} / \mathrm{L}$, increase in $\left[\mathrm{Na}^{+}\right] \geq$ $5 \mathrm{mmol} / \mathrm{L}$ from baseline, switch to another therapy, discharge with persistent $\mathrm{HN}$, or death or transfer to hospice with persistent $\mathrm{HN})$. The first $\left[\mathrm{Na}^{+}\right]$obtained on the day after treatment was discontinued and was then used as the endof-therapy value. Patients in whom $\left[\mathrm{Na}^{+}\right]$corrected to $>$ $130 \mathrm{mmol} / \mathrm{L}$ with initial therapy were assessed for relapse of $\mathrm{HN}$ during the subsequent hospitalization. Patients who were switched by the treating physician to a different treatment were assessed and reclassified based on second treatment provided in the same manner as that for the initial therapy.

At hospital discharge, final $\left[\mathrm{Na}^{+}\right]$and disposition (discharge home with corrected or persistent $\mathrm{HN}$, and mortality [hospital death or transfer to hospice]) were recorded, and LOS was determined. For patients in whom discharge was delayed due to nonmedical reasons, the additional days were not included in LOS if documented as such in the clinical record.

2.2. Statistical Analysis. Clinical characteristics, initial and final $\left[\mathrm{Na}^{+}\right]$, hospital mortality, and median LOS of patients with $\mathrm{HN}$ at hospital admission versus those who developed $\mathrm{HN}$ during hospitalization were compared. The relationship between HN severity and the various clinical parameters, LOS, and hospital mortality were assessed for patients admitted with HN.

Characteristics were compared among the various treatment groups. Cumulative endpoint outcomes were recorded for Days 1-5 and final outcomes at the end of primary treatment. The percentage of patients with initial $[\mathrm{Na}]<125$ in whom the level increased by $\geq 5 \mathrm{mmol} / \mathrm{L}$ on Days 2 and 3 and at the end of therapy was assessed. For patients with moderate or severe $\mathrm{HN}$, the percentage of patients with an increase in $\left[\mathrm{Na}^{+}\right] \geq 5 \mathrm{mmol} / \mathrm{L}$ was assessed on Days 2 and 3 and end of treatment. A similar analysis was performed for patients who received a secondary therapy. For patients in whom $\left[\mathrm{Na}^{+}\right]$ corrected to $>130 \mathrm{mmol} / \mathrm{L}$ with initial therapy, characteristics and LOS were compared between patients who did and did not experience a relapse.

Descriptive statistics for continuous variables consisted of median number of observations and interquartile range (IQR). Frequency counts and percentages were obtained for categorical variables. Statistical comparisons of continuous variables were performed using nonparametric tests such as the Wilcoxon rank-sum test. Comparisons of categorical variables were performed using chi-square tests for association. Statistical significance for the tests was defined at the 5\% level $(P<0.05)$.

2.3. Internal Review Board Approval. Approval was sought from the local research ethics review board at each site using either informed consent or a waiver of consent.

\section{Results}

3.1. Patient Characteristics. Of the 3087 patients who satisfied the inclusion and exclusion criteria, $650(21 \%)$ had cirrhosis and 595 met the criteria for the current analysis. Baseline characteristics are presented in Table 1 . HN was associated with advanced liver disease and severe portal hypertension (Table 2). HN was present in 518 patients (87\%) on admission and developed during hospitalization in 77 (13\%). Patients with $\mathrm{HN}$ on admission had lower initial $\left[\mathrm{Na}^{+}\right]$, higher blood urea nitrogen (BUN), and MELD-Na score $(P<0.05$; Table 2$)$. More than half of the patients had large-volume ascites (Supplemental Table 1). Patients with moderate $(25 \% ; P<$ $0.05)$ or severe $\mathrm{HN}(28 \% ; P<0.05)$ more commonly had overt HE than those with mild HN (17\%).

3.2. Initial HN Treatment. The most common initial therapies were NS (36\%), FR (33\%), NST (20\%), TO (5\%), and HS (2\%; Supplemental Table 2). A variety of other therapies (e.g., salt tablets and conivaptan) and combinations were administered to 22 patients (4\%). Initial $\left[\mathrm{Na}^{+}\right]$in the NST group was higher than in the other groups $(P<0.05)$. Initial $\left[\mathrm{Na}^{+}\right]$in the FR 
TABLE 1: Baseline demographic characteristics.

\begin{tabular}{|c|c|c|}
\hline & $\begin{array}{l}\text { All Patients }^{\mathrm{i}} \\
(\mathrm{N}=3,087)\end{array}$ & $\begin{array}{l}\text { Cirrhosis } \\
(\mathrm{n}=630)\end{array}$ \\
\hline \multicolumn{3}{|l|}{ Age distribution, $\mathrm{n}(\%)^{\mathrm{a}}$} \\
\hline$\leq 50 \mathrm{y}$ & $479(16)$ & $190(30)$ \\
\hline $51-64$ y & $937(30)$ & $339(54)$ \\
\hline $65-74$ y & $587(19)$ & $81(13)$ \\
\hline$\geq 75 y$ & $1,084(35)$ & $20(3)$ \\
\hline Men, $\mathrm{n}(\%)^{\mathrm{b}}$ & $1,558(51)$ & $419(67)$ \\
\hline \multicolumn{3}{|l|}{ Race distribution: US only, $\mathrm{n}(\%)^{\mathrm{a}}$} \\
\hline White & $1,927(74)$ & $455(72)$ \\
\hline African-American & $309(12)$ & $58(9)$ \\
\hline Asian & $57(2)$ & $13(2)$ \\
\hline Other & $154(6)$ & $53(9)$ \\
\hline Unknown & $149(6)$ & $51(8)$ \\
\hline Mean initial $[\mathrm{Na}+] \pm \mathrm{SD}, \mathrm{mEq} / \mathrm{L}^{\mathrm{c}}$ & $123.6 \pm 5.5$ & $124.1 \pm 5.0$ \\
\hline Mean initial $\mathrm{BUN} \pm \mathrm{SD}, \mathrm{mg} / \mathrm{dL}^{\mathrm{a}}$ & $20.8 \pm 16.8$ & $25.5 \pm 18.8$ \\
\hline Mean initial creatinine $\pm S D, m g / \mathrm{dL}^{\mathrm{d}}$ & $1.1 \pm 0.73$ & $1.28 \pm 0.85$ \\
\hline Initial BUN:creatinine ratio $^{a}$ & $19.4 \pm 9.4$ & $19.8 \pm 8.6$ \\
\hline \multicolumn{3}{|l|}{ Prior $\mathrm{HN}, \mathrm{n}(\%)^{\mathrm{a}, \mathrm{e}}$} \\
\hline Yes & $909(29)$ & $240(38)$ \\
\hline No & $1,176(38)$ & $178(28)$ \\
\hline Unknown & $1,001(32)$ & $212(34)$ \\
\hline \multicolumn{3}{|l|}{$\overline{\mathrm{HN}}$ at admission, $\mathrm{n}(\%)^{\mathrm{f}}$} \\
\hline Yes & $2,532(82)$ & $549(87)$ \\
\hline No & $531(17)$ & $81(13)$ \\
\hline Unknown & $24(1)$ & $0(0)$ \\
\hline \multicolumn{3}{|l|}{ Primary physician specialty, n (\%) } \\
\hline Nephrologist & $104(3)$ & $8(1)$ \\
\hline Endocrinologist & $108(4)$ & 0 \\
\hline Cardiologist & $321(10)$ & $7(1)$ \\
\hline Hepatologist & $260(8)$ & $246(39)$ \\
\hline Oncologist & $111(4)$ & $11(2)$ \\
\hline Generalist & $1,844(60)$ & $315(50)$ \\
\hline Other & $338(11)$ & $43(7)$ \\
\hline \multicolumn{3}{|l|}{ HN subspecialist consulted, $\mathrm{n}(\%)^{\mathrm{g}, \mathrm{h}}$} \\
\hline No & $1989(64)$ & $501(80)$ \\
\hline Yes & $1,096(36)$ & $129(21)$ \\
\hline
\end{tabular}

Abbreviations: BUN, blood urea nitrogen; CHF, congestive heart failure; $\mathrm{HN}$, hyponatremia; $\left[\mathrm{Na}^{+}\right]$, sodium concentration; SD, standard deviation; SIADH, syndrome of inappropriate antidiuretic hormone secretion.

${ }^{a}$ SIADH vs CHF and cirrhosis, and CHF vs cirrhosis: $P<0.001$.

${ }^{\mathrm{b}} \mathrm{SIADH}$ vs CHF: $P=0.79$; and SIADH and CHF vs cirrhosis: $P<0.001$.

${ }^{\circ} \mathrm{SIADH}$ vs CHF and cirrhosis: $P<0.001$; CHF vs cirrhosis: $P=0.01$.

${ }^{\mathrm{d}}$ SIADH vs CHF and cirrhosis: $P<0.001$; and CHF vs cirrhosis: $P=0.05$.

${ }^{\mathrm{e}} \mathrm{HN}$ during previous hospital admission in prior 12 months.

${ }^{\mathrm{f}}$ Data missing for 24 patients in All, 19 in SIADH, and 4 in CHF populations; SIADH vs CHF: $P=0.04$; SIADH vs cirrhosis: $P=0.001$; and CHF vs cirrhosis: $P<0.001$.

${ }^{g}$ SIADH vs CHF and cirrhosis: $P<0.001$; and CHF vs cirrhosis: $P=0.01$.

${ }^{\mathrm{h}} \mathrm{HN}$ specialist defined as nephrologist or endocrinologist.

${ }^{\mathrm{i}}$ Includes 171 patients without a diagnosis of SIADH, cirrhosis, or CHF. 
TABLE 2: Clinical characteristics of cirrhosis patients admitted with HN subdivided by hyponatremia severity.

\begin{tabular}{|c|c|c|c|c|}
\hline & $\begin{array}{c}\text { Total } \\
\mathrm{N}=518\end{array}$ & $\begin{array}{c}<120 \\
\mathrm{n}=106\end{array}$ & $\begin{array}{c}\left.\mathrm{Ta}^{+}\right], \mathrm{mmol} / \mathrm{L} \\
\geq 120-\leq 125 \\
\mathrm{n}=202\end{array}$ & $\begin{array}{c}>125-\leq 130 \\
\mathrm{n}=210 \\
\end{array}$ \\
\hline Median age, $y$ & 56 & 54 & 56 & 57 \\
\hline Male/female, $\mathrm{n}$ & $345 / 173$ & $73 / 33$ & $130 / 72$ & $142 / 68$ \\
\hline BUN, mg/dL & $20.0(19.0)$ & $18.0(20.5)$ & $21.0(20.0)$ & $19.0(17.0)$ \\
\hline $\mathrm{Cr}, \mathrm{mg} / \mathrm{dL}$ & $1.0(0.6)$ & $1.0(0.7)$ & $1.1(0.7)$ & $1.0(0.6)$ \\
\hline BUN:Cr ratio & $18.9(10.4)$ & $19.0(12.0)$ & $19.8(11.6)$ & $18.0(9.1)$ \\
\hline Alb, g/dL & $2.5(0.8)$ & $2.6(1.1)$ & $2.5(0.8)$ & $2.4(0.6)$ \\
\hline Tbili, $\mu \mathrm{mol} / \mathrm{L}$ & $4.3(7.4)$ & $4.3(7.2)$ & $4.5(6.9)$ & $4.3(7.6)$ \\
\hline INR, s & $1.7(0.6)$ & $1.6(0.5)$ & $1.7(0.7)$ & $1.7(0.7)$ \\
\hline Severe ascites, $\mathrm{n}(\%)$ & $284(55)$ & $53(50)$ & $124(61)$ & $107(51)$ \\
\hline Severe HE, n (\%) & $116(22)$ & $30(28)$ & $51(25)$ & $35(17)$ \\
\hline C-P score & $11.0(3.0)$ & $10.5(3.0)$ & $11.0(3.0)$ & $10.0(3.0)$ \\
\hline MELD score & $20.2(9.7)$ & $18.7(9.0)$ & $20.8(8.0)$ & $20.2(10.4)$ \\
\hline MELD-Na score & $27.3(6.3)$ & $26.7(5.6)$ & $28.0(5.0)$ & $26.3(7.6)$ \\
\hline
\end{tabular}

${ }^{\mathrm{a}} P<0.01$.

Values for blood urea nitrogen (BUN), creatinine (Cr), BUN:Cr ratio, albumin (Alb), total bilirubin (Tbili), international normalized ratio (INR), and Child-Pugh (CP), Model for End-Stage Liver Disease (MELD), and MELD-NA scores are median (interquartile range). HE, hepatic encephalopathy; HN, hyponatremia.

group was higher than in the HS and TO groups (both $P<$ 0.05). $\left[\mathrm{Na}^{+}\right]$increased at greater rates in the FR versus NST group $(P=0.03)$, NS versus NST and FR groups $(P<0.05)$, and HS and TO versus NST and FR groups $(P<0.05$ for all). Median length of treatment for NST, FR, and TO was 3 days and 2 days for NS and HS. It is important to note that the goal of this observational study was to demonstrate the current state of treatment management of hypervolemic $\mathrm{HN}$ in various real-world hospital settings. The duration of therapy and the treatment choice were determined by the treating physician as indicated in the clinical chart.

Figure 1 presents the response to initial treatment by various HN categories at Days 1-5. The percentages of patients with moderate or severe $\mathrm{HN}$ were significantly higher in the HS $(82 \%)$ and TO (78\%) groups than in the NS (65\%) and FR $(63 \%)$ groups, which, in turn, were higher than in the NST group (32\%; all $P<0.05)$. Patients in the HS or TO groups more frequently improved into more less severe $\mathrm{HN}$ or treatment success categories than in those treated with NST, FR, or NS.

Table 3 presents the percentages of patients with moderate or severe $\mathrm{HN}$ in which $\left[\mathrm{Na}^{+}\right]$increased by $\geq 5 \mathrm{mmol} / \mathrm{L}$ at Days 2 and 3 and at final outcome. In the NST and FR groups, $27 \%$ of patients achieved this endpoint at Day 2 and $33 \%$ and $36 \%$, respectively, at Day 3. Higher percentages achieved this endpoint in the NS versus NST and FR groups at Day $2(P=$ 0.08 and $=0.02$, respectively) and Day $3(P=0.08$ and $<0.01)$. There was a more rapid response in the HS versus NST and FR groups (Days 2 and 3, $P<0.03$ for both) and TO versus NST and FR groups (Day $2[P=0.15$ and 0.07 , respectively] and Day $3[P<0.01$ for both]). The percentages of patients with treatment success were significantly higher in the TO versus NST, FR, and NS groups, and HS versus FR group (all $P<$ 0.05).
Of patients admitted to the hospital with HN, 151 (29\%) were not receiving diuretic therapy prior to hospital admission. Among these patients, $34(23 \%)$ received $\geq 1$ dose of a diuretic during initial HN therapy. Of 367 patients $(71 \%)$ who received diuretics prior to hospital admission, 287 (78\%) received $\geq 1$ dose of a diuretic during initial $\mathrm{HN}$ therapy.

3.3. Secondary HN Treatment. A second therapy was provided to 275 patients. The secondary $H N$ treatments based on initial therapy, and the characteristics and outcomes by secondary treatment group are presented in Supplemental Tables 4 and 5. Sodium levels prior to the secondary therapy and response rates are presented in Table 3. In general, the sodium response was similar for a specific therapy regardless of whether it was administered as initial or secondary therapy.

3.4. HN Relapse and Final Outcomes. Of the 110 patients who corrected with initial therapy, 61 (55\%) experienced a relapse of $\mathrm{HN}$ during the subsequent hospitalization (Table 4). Characteristics, initial and final $\left[\mathrm{Na}^{+}\right]$levels, and LOS until $\mathrm{HN}$ correction were comparable between patients who did and did not relapse. The LOS after HN correction (6 versus 2 days) and total LOS (9 versus 6 days) were higher in patients who relapsed versus those patients whose $[\mathrm{Na}]$ remained above $130(P<0.05)$.

For patients admitted with $\mathrm{HN}$, final median $\left[\mathrm{Na}^{+}\right]$was 129 (IQR 7) mmol/L. Of these patients, 174 (34\%) were discharged alive with corrected HN, 292 (56\%) were discharged with persistent $\mathrm{HN}$ (mild: 203 [39\%]; moderate: 84 [16\%]; and severe: $5[1 \%])$, and $49(10 \%)$ died during hospitalization or were discharged to hospice. For patients who developed HN during hospitalization, final median $\left[\mathrm{Na}^{+}\right]$was 130 (IQR 6) $\mathrm{mmol} / \mathrm{L}$. Of these patients, $25(33 \%)$ were discharged alive with corrected HN, 39 (51\%) were discharged with persistent 
TABLE 3: Patients with $\left[\mathrm{Na}^{+}\right] \geq 5 \mathrm{mmol} / \mathrm{L}$ in response to initial and secondary therapy ${ }^{\mathrm{a}}$.

\begin{tabular}{|c|c|c|c|}
\hline & $\begin{array}{c}\text { Day } 2 \\
\text { Response }\end{array}$ & $\begin{array}{c}\text { Day } 3 \\
\text { response }\end{array}$ & Final response \\
\hline \multicolumn{4}{|c|}{ Initial therapy, $\mathrm{n}(\%)$} \\
\hline NST & $9(27)$ & $11(33)$ & $13(39)$ \\
\hline FR & $29(27)$ & $38(36)$ & $42(39)$ \\
\hline NS & $54(45)$ & $61(51)$ & $62(52)$ \\
\hline HS & $7(78)$ & $7(78)$ & $7(78)$ \\
\hline TO & $10(48)$ & $15(71)$ & $17(81)$ \\
\hline \multicolumn{4}{|c|}{ Secondary therapy, n (\%) } \\
\hline NST & $5(36)$ & $5(36)$ & $5(36)$ \\
\hline FR & $15(31)$ & $18(37)$ & $18(37)$ \\
\hline NS & $9(29)$ & $10(32)$ & $10(32)$ \\
\hline HS & $10(100)$ & $10(100)$ & $10(100)$ \\
\hline TO & $15(58)$ & $17(65)$ & $18(68)$ \\
\hline
\end{tabular}

${ }^{\mathrm{a}}$ Patients with initial moderate or severe hyponatremia.

Initial therapy $P<0.05$ for Day 2 response: no specific therapy (NST) vs hypertonic saline (HS), and fluid restriction (FR) vs HS and isotonic saline (NS); Day 3 response: NST vs HS and tolvaptan (TO); FR vs HS, NS, and TO: final response: NST vs TO, FR vs HS and TO, and NS vs TO.
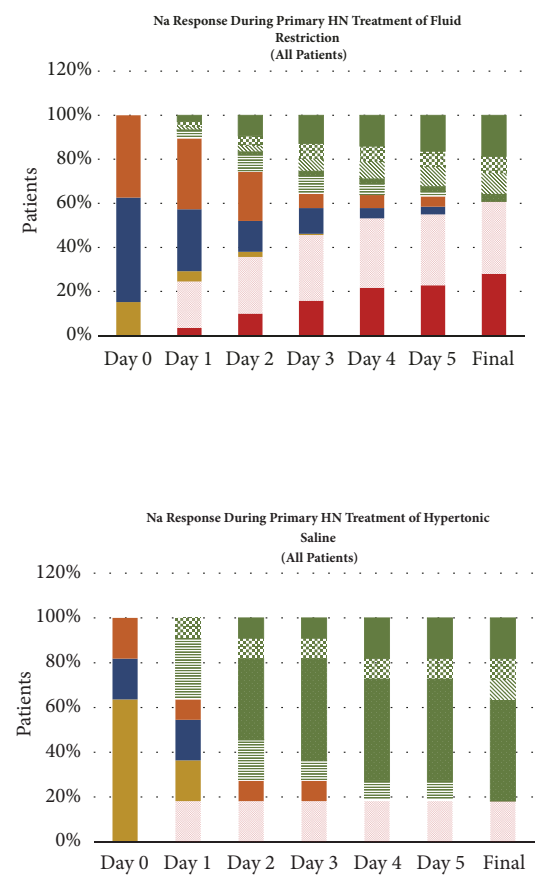

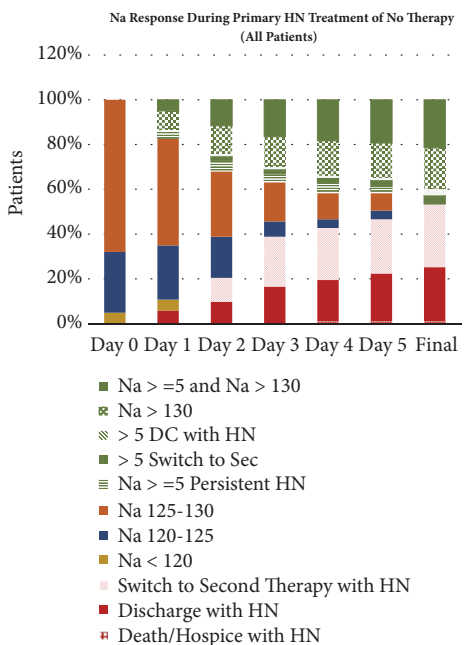

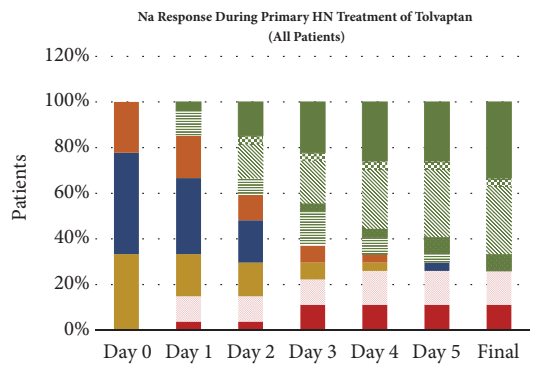

FIGURE 1: Response to primary hyponatremia $(\mathrm{HN})$ treatment by various $\mathrm{HN}$ categories. $\left[\mathrm{Na}^{+}\right]$, sodium concentration; FR, fluid restriction; NS, isotonic saline; NST, no specified therapy; HS, hypertonic saline; TO, tolvaptan; DC, discontinuation.

HN (mild: 31 [40\%]; moderate: 8 [10\%]; and severe: 0 ), and $12(16 \%)$ died during hospitalization or were discharged to hospice.

Median (IQR) LOS values for patients admitted with $\mathrm{HN}$ and those who developed $\mathrm{HN}$ during hospitalization were 6 (5) and 9 (8), respectively $(P<0.05)$. The distribution of LOS for patients admitted with HN versus those who developed HN during hospitalization is presented in Figure 2. Of patients admitted with $\mathrm{HN}$ compared with those who developed $\mathrm{HN}$ during hospitalization, $90 \%$ versus $75 \%$ were discharged by 14 days. Hospital mortality was numerically greater in patients who developed $\mathrm{HN}$ during hospitalization than in those admitted with HN but not statistically significant $(16 \%$ versus $10 \% ; P=0.25)$.

\section{Discussion}

This analysis of the HN Registry-the largest observational study to specifically examine $\mathrm{HN}$ in the hospital setting-produced several important findings. There was a 
TABle 4: Patient characteristics and LOS for patients admitted with HN who corrected with initial HN therapy.

\begin{tabular}{lcc}
\hline & Relapse $(\mathrm{n}=61)$ & No relapse $(\mathrm{n}=49)$ \\
\hline$\left[\mathrm{Na}^{+}\right], \mathrm{mmol} / \mathrm{L}$ & $128.0(4.0)$ & $127.0(5.0)$ \\
{$\left[\mathrm{Na}^{+}\right]$at time of correction, $\mathrm{mmol} / \mathrm{L}^{\mathrm{a}}$} & $132.0(2.0)$ & $132.0(2.0)$ \\
$\mathrm{BUN}, \mathrm{mg} / \mathrm{dL}$ & $17.0(17.0)$ & $16.0(16.0)$ \\
$\mathrm{Cr}, \mathrm{mg} / \mathrm{dL}$ & $1.0(0.5)$ & $1.0(0.9)$ \\
$\mathrm{BUN}$ Cr ratio & $19.1(9.6)$ & $17.4(11.4)$ \\
$\mathrm{Tbili}, \mu \mathrm{mol} / \mathrm{L}$ & $4.8(7.3)$ & $4.3(6.7)$ \\
$\mathrm{INR}, \mathrm{s}$ & $1.8(0.8)$ & $1.8(0.5)$ \\
Alb, $\mathrm{g} /$ doll & $2.6(0.9)$ & $2.8(0.7)$ \\
$\mathrm{MELD}$ score at correction & $20.9(6.6)$ & $21.9(11.3)$ \\
LOS, d & $9(6.0)$ & $6(5.0)$ \\
LOS until correction, $\mathrm{d}$ & $3(2.0)$ & $3(1.0)$ \\
LOS after correction, $\mathrm{d}^{\mathrm{a}}$ & $6(5.0)$ & $2(3.0)$ \\
Death/ hospice, $\mathrm{n}(\%)$ & $5(8)$ & $5(10)$ \\
\hline$<0.05$, & & \\
\hline
\end{tabular}

a $<0.05$.

Values for sodium concentration $\left(\left[\mathrm{Na}^{+}\right]\right),\left[\mathrm{Na}^{+}\right]$at time of correction, blood urea nitrogen (BUN), creatinine $(\mathrm{Cr}), \mathrm{BUN}$ :CR ratio, total bilirubin (Tbili), albumin (Alb), international normalized ratio (INR), Model for End-Stage Liver Disease (MELD) score at correction, length of stay (LOS), LOS until correction, and LOS after correction are median (interquartile range). HN, hyponatremia.

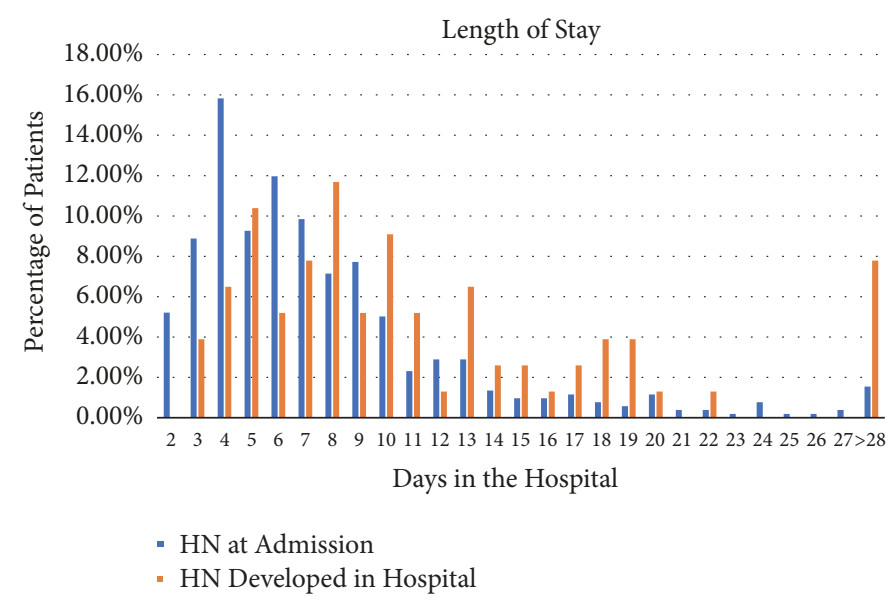

FIGURE 2: The distribution of length of stay (LOS) for patients with hyponatremia (HN) at admission versus those who developed HN during hospitalization.

strong association between $\mathrm{HN}$ and advanced cirrhosis with severe portal hypertension as has been previously reported [3]. Large-volume ascites and overt HE were noted in 54\% and $22 \%$ of patients admitted with $\mathrm{HN}$, respectively. There was not a relationship between the presence of severe ascites and the severity of $\mathrm{HN}(\mathrm{p}=0.216)$. However, the prevalence of overt $\mathrm{HE}$ was related to HN severity.

Treatment approaches were highly variable and frequently ineffective [7]. Twenty percent of patients received NST, and only $34 \%$ of the cirrhotic patients admitted to the hospital with $\mathrm{HN}$ were discharged with corrected $\mathrm{HN}$. Although FR is recommended in treatment guidelines as initial therapy [10], this is the first study to report outcomes in patients treated with FR in real life practice. The rate of increase in $\left[\mathrm{Na}^{+}\right]$with FR treatment was of limited efficacy and comparable to NST. Of patients with moderate and severe $\mathrm{HN},\left[\mathrm{Na}^{+}\right]$increased by $\geq 5 \mathrm{mmol} / \mathrm{L}$ in only $27 \%$ and $36 \%$ at Days 2 and 3, respectively. These results are in accordance with previous studies that showed a limited or no response with FR $[11,12]$.

Although not recommended, treatment with NS was the most common initial therapy [13]. Ascites is frequently the reason for hospitalization, and NS can exacerbate its severity and increase the need for invasive procedures, such as paracentesis. It may also exacerbate HN through "a desalination process" in which increased AVP levels lead to water retention and excretion of hypertonic urine [14]. Patients receiving NS had lower $\left[\mathrm{Na}^{+}\right]$than those receiving FR. Although the rate of increase in $\left[\mathrm{Na}^{+}\right]$was greater, its effectiveness was limited: of patients with moderate or severe $\mathrm{HN},\left[\mathrm{Na}^{+}\right]$only increased by $\geq 5 \mathrm{mmol} / \mathrm{L}$ in $45 \%$ and $51 \%$ of patients at Days 2 and 3 , respectively.

Correction of HN was most effective with HS and TO. HS is recommended for severe symptomatic $\mathrm{HN}$ and was used 
as initial therapy in $2 \%$ of patients [15]. $\left[\mathrm{Na}^{+}\right]$was lowest in patients receiving $\mathrm{HS}$, and $82 \%$ had either moderate or severe HN. There are currently no reports on the response to HS in cirrhosis. In this study, HS led to a rapid increase in $\left[\mathrm{Na}^{+}\right]$- an increase $\geq 5 \mathrm{mmol} / \mathrm{L}$ by Day 2 in $78 \%$ of patients.

TO was used as primary therapy in $5 \%$ of patients. The severity of $\mathrm{HN}$ was similar to those receiving HS, and $78 \%$ patients had either moderate or severe $\mathrm{HN}$. As with $\mathrm{HS},\left[\mathrm{Na}^{+}\right]$ rapidly increased but at a slightly more gradual rate. At Day 2 , $48 \%$ of patients had an increase $\geq 5 \mathrm{mmol} / \mathrm{L}$. However, the percentage at Day 3 was comparable to HS (71\% versus $78 \% ; P=1.00)$. This response rate was comparable to that observed in the cirrhosis population from the SALT1/SALT2 trials in which $40 \%$ and $70 \%$ of patients treated with TO had an increase $\geq 5 \mathrm{mmol} / \mathrm{L}$ at Days 2 and 3 , respectively (data on file).

$\mathrm{HN}$ is frequent in patients with large-volume ascites [3]. In addition to the nonosmotic release of AVP, the reninaldosterone system is activated, leading to increased renal sodium reabsorption. Diuretics are the mainstay of treatment of fluid overload but can exacerbate $\mathrm{HN}$ by decreasing intravascular volume (leading to increased AVP release) and only blocking sodium reabsorption, leaving continued freewater absorption unopposed [16]. Guidelines recommend tapering and then discontinuing diuretics if $\mathrm{HN}$ persists despite FR. However, diuretics were discontinued in only $17 \%$ of patients with $\mathrm{HN}$ at admission and were initiated in $7 \%$ of patients.

$\mathrm{HN}$ is associated with increased LOS in hospitalized patients [17-19]. In this study the impact of HN relapse in LOS after initial sodium correction was especially striking. Despite comparable initial $\mathrm{Na}$ level, level at correction, and time to correction, LOS was 4 days longer in those in whom $\mathrm{HN}$ recurred.

The results of this study are limited by its observational nature and broad definitions. FR was defined only by the physician orders indicated in the chart. NS and diuretic administration were also broadly defined. Albumin administration which is increasingly being used for the treatment of $\mathrm{HN}$ and infectious complications were not assessed [20]. In addition, $\mathrm{Na}$ levels have recently been reported to increase in response to treatment with midodrine and octreotide in a noncontrolled study [21]. However, the goal of this study was to evaluate treatment practices and outcomes in the real-world setting with the most commonly used approaches. The frequent administration of ineffective and/or nonstandardized therapy that frequently includes NS is not consistent with treatment guidelines. Correction of only $34 \%$ of patients at discharge suggests that most physicians do not view correction of $\mathrm{HN}$ as a meaningful clinical endpoint. Correction of $\mathrm{HN}$ is less important than demonstration of a beneficial impact on clinical endpoints. A question that invariably arises is whether $\mathrm{HN}$ is a direct participant in the pathophysiologic process and directly contributes to poor outcomes and increased LOS, or whether it is only a marker of end-stage disease. Preliminary evidence supports a contributory role for $\mathrm{HN}$ in hepatic encephalopathy [22]. Finally, treatment of $\mathrm{HN}$ with $\mathrm{TO}$ has also been shown to shorten LOS in patients with heart failure, syndrome of inappropriate diuretic hormone, and cancer [23, 24].

Determination of the impact of the treatment of $\mathrm{HN}$ in patients with cirrhosis will first require standardization of its management with effective therapy. The initial use of FR should be reevaluated, while NS administration should be avoided. Although HS is effective, it is limited by its deleterious impact on fluid overload and need for close monitoring, which frequently requires an intensive-care setting. In addition, the development of tense ascites due to HS administration can aggravate the severity of portal hypertension $[23,24]$. Early treatment with TO offers an effective approach that should allow comprehensive assessment of the importance of $\mathrm{HN}$ treatment in the hospitalized patient with cirrhosis. Because the FDA-approved indications for TO have removed cirrhosis as an approved population, it is important that treatment with TO in these patients be performed in a carefully controlled manner.

\section{Data Availability}

This was an observational study, not a randomized, controlled clinical trial, supported by Otsuka America Pharmaceutical, Inc. All statistical analyses were performed by an outside organization, Mapi Group, using predefined definitions and independent of Otsuka. In the event there were issues, they were adjudicated by 2 members of the Hyponatrema Registry Steering Committee. We would be happy to answer any additional questions. As indicated in the Methods section, all statistical analyses were performed by MAPI using predefined criteria. MAPI is an independent biostatistical organization that is routinely employed for independent analysis of data sets. All analyses were performed independently of investigator in-put. The data for each patient is present in an elaborate Excel spreadsheet that requires detailed explanation in its use.

\section{Ethical Approval}

The study was reviewed and approved by each site's respective Institutional Review Board.

\section{Consent}

Depending on the local site requirements, study participants, or their legal guardian, provided informed written consent prior to study enrollment. Because the study was observational only and treatment was provided based on routine clinical practice of the patient's treating physician, selected sites permitted enrollment with a waiver of consent.

\section{Conflicts of Interest}

Samuel H. Sigal is a consultant, has received travel support and fees for data review, and has served on a speakers' bureau for Otsuka. His institution received research support for serving as an investigational site for the Hyponatremia Registry and for an investigator-initiated trial from Otsuka. Alpesh Amin is a consultant for the Hyponatremia Registry, 
has received travel support and fees for data review activities, and has served on a speakers' bureau for Otsuka. Joseph A. Chiodo III was employed by Otsuka America Pharmaceutical, Inc. at the time the manuscript was developed. Arun Sanyal is President of Sanyal Biotechnology and has stock options in Genfit, Akarna, Tiziana, Indalo, Durect. He has served as a consultant to Hemoshear, Echosens, AbbVie, Astra Zeneca, Nitto Denko, Ardelyx, Conatus, Nimbus, Amarin, Salix, Tobira, Takeda, Novo Nordisk, Fibrogen, Jannsen, Gilead, Boehringer, Lilly, Zafgen, Novartis, Pfizer, Immuron, Exhalenz, and Genfit. He has been an unpaid consultant to Intercept, Immuron, Galectin, Fractyl, Syntlogic, Affimune, Chemomab, Nordic Bioscience, and Bristol Myers Squibb. His institution has received grant support from Gilead, Salix, Tobira, Bristol Myers, Shire, Echosens, Intercept, Merck, Astra Zeneca, Malinckrodt, Cumberland, and Novartis. He receives royalties from Elsevier and UptoDate.

\section{Authors' Contributions}

Samuel H. Sigal participated in data acquisition and analysis and wrote the paper. Alpesh Amin and Arun Sanyal participated in data acquisition and analysis and manuscript preparation. Joseph A. Chiodo III participated in data analysis and manuscript preparation.

\section{Acknowledgments}

This work was supported by Otsuka America Pharmaceutical, Inc. Jamie Jarecki-Smith of Clinovative Research provided continuing support for reviewing data entered in preparation for analysis. Statistical analysis was performed by the Mapi ${ }^{\mathrm{TM}}$ Group, Lexington, KY, USA, with funding by Otsuka. Editorial assistance was provided by Catherine Fontana and Geoff Marx of BioScience Communications, New York, NY, USA, which was funded by Otsuka.

\section{Supplementary Materials}

Supplemental Table 1: this table provides the clinical characteristics, LOS, and mortality of cirrhotic patients in the Hyponatremia Registry. It includes a breakdown between those who presented with $\mathrm{HN}$ at hospital admission versus those who developed HN during the hospital stay. Supplemental Table 2: this table shows the clinical characteristics including $\mathrm{HN}$ severity of the cirrhotic patients with $\mathrm{HN}$ at admission based on initial therapy selected by the treating physician. Supplemental Table 3: this table presents the outcomes of the initial therapies of cirrhotic patients admitted with $\mathrm{HN}$, including the percentages switched to a second therapy, discharged with persistent HN, mortality, and length of stay. Supplemental Table 4: this table provides the outcomes of the secondary therapies of the patients who presented with $\mathrm{HN}$ and did not respond to the initial therapy. The initial treatment is indicated in the far-left column and the secondary therapy is indicated in the top row. Supplemental Table 5: this table presents the outcomes of the secondary therapies of cirrhotic patients who were admitted with $\mathrm{HN}$ and did not respond to the initial therapy. (Supplementary Materials)

\section{References}

[1] Y. Iwakiri and R. J. Groszmann, "The hyperdynamic circulation of chronic liver diseases: From the patient to the molecule," Hepatology, vol. 43, no. 2, pp. S121-S131, 2006.

[2] P. Ginès and M. Guevara, "Hyponatremia in cirrhosis: Pathogenesis, clinical significance, and management," Hepatology, vol. 48, no. 3, pp. 1002-1010, 2008.

[3] P. Angeli, F. Wong, H. Watson et al., "Hyponatremia in cirrhosis: Results of a patient population survey, Hepatology, vol. 44, no. 6, pp. 1535-1542, 2006.

[4] S. Deitelzweig, A. Amin, R. Christian, K. Friend, J. Lin, and T. J. Lowe, "Hyponatremia-associated healthcare burden among us patients hospitalized for cirrhosis," Advances in Therapy, vol. 30, no. 1, pp. 71-80, 2013.

[5] Vaprisol, "Nashville, TN: Cumberland Pharmaceuticals, Inc," 2014.

[6] Samsca, “Tokyo, Japan: Otsuka Pharmaceutical Co, Ltd, 2012.

[7] P. B. Watkins, J. H. Lewis, N. Kaplowitz et al., "Clinical pattern of tolvaptan-associated liver injury in subjects with autosomal dominant polycystic kidney disease: analysis of clinical trials database," Drug Safety, vol. 38, no. 11, pp. 1103-1113, 2015.

[8] A. Greenberg, J. G. Verbalis, A. N. Amin et al., "Current treatment practice and outcomes. Report of the hyponatremia registry," Kidney International, vol. 88, no. 1, pp. 167-177, 2015.

[9] P. S. Kamath and W. R. Kim, "The model for end-stage liver disease (MELD)," Hepatology, vol. 45, no. 3, pp. 797-805, 2007.

[10] J. G. Verbalis, S. R. Goldsmith, A. Greenberg et al., "Diagnosis, evaluation, and treatment of hyponatremia: expert panel recommendations," American Journal of Medicine, vol. 126, no. 10, supplement 1, pp. S1-S42, 2013.

[11] F. Wong, A. T. Blei, L. M. Blendis, and P. J. Thuluvath, "A vasopressin receptor antagonist (VPA-985) improves serum sodium concentration in patients with hyponatremia: A multicenter, randomized, placebo-controlled trial," Hepatology, vol. 37, no. 1, pp. 182-191, 2003.

[12] A. L. Gerbes, V. Gülberg, P. Ginès et al., "Therapy of hyponatremia in cirrhosis with a vasopressin receptor antagonist: A randomized double-blind multicenter trial," Gastroenterology, vol. 124, no. 4, pp. 933-939, 2003.

[13] European Association for the Study of the Liver, "EASL clinical practice guidelines on the management of ascites, spontaneous bacterial peritonitis, and hepatorenal syndrome in cirrhosis," Journal of Hepatology, vol. 53, no. 3, pp. 397-417, 2010.

[14] A. Steele, M. Gowrishankar, S. Abrahamson, C. D. Mazer, R. D. Feldman, and M. L. Halperin, "Postoperative hyponatremia despite near-isotonic saline infusion: a phenomenon of desalination," Annals of Internal Medicine, vol. 126, pp. 20-25, 1997.

[15] S. John and P. J. Thuluvath, "Hyponatremia in cirrhosis: pathophysiology and management," World Journal of Gastroenterology, vol. 21, no. 11, pp. 3197-3205, 2015.

[16] G. Liamis, H. Milionis, and M. Elisaf, "A review of drug-induced hyponatremia," American Journal of Kidney Diseases, vol. 52, no. 1, pp. 144-153, 2008.

[17] A. Amin, S. Deitelzweig, R. Christian et al., "Evaluation of incremental healthcare resource burden and readmission rates associated with hospitalized hyponatremic patients in the US," Journal of Hospital Medicine, vol. 7, no. 8, pp. 634-639, 2012. 
[18] A. Amin, S. Deitelzweig, R. Christian, K. Friend, J. Lin, and T. J. Lowe, "Healthcare resource burden associated with hyponatremia among patients hospitalized for heart failure in the US," Journal of Medical Economics, vol. 16, no. 3, pp. 415420, 2013.

[19] R. Berardi, M. Caramanti, M. Castagnani et al., "Hyponatremia is a predictor of hospital length and cost of stay and outcome in cancer patients," Supportive Care in Cancer, vol. 23, no. 10, pp. 3095-3101, 2015.

[20] A. Garioud, J. Cadranel, A. Pauwels et al., "Albumin use in patients with cirrhosis in France: results of the 'ALBU-LIVE' survey: a case for better EASL guidelines diffusions and/or revision," Journal of Clinical Gastroenterology, vol. 51, pp. 831838, 2017.

[21] S. Patel, D. Nguyen, A. Rastogi, M. Nguyen, and M. K. Nguyen, "Treatment of cirrhosis-associated hyponatremia with midodrine and octreotide," Frontiers in Medicine, vol. 4, 2017.

[22] V. Ahluwalia, D. M. Heuman, G. Feldman et al., "Correction of hyponatraemia improves cognition, quality of life, and brain oedema in cirrhosis," Journal of Hepatology, vol. 62, no. 1, pp. 75-82, 2016.

[23] J. R. Chiong, S. Kim, J. Lin, R. Christian, and J. F. Dasta, "Evaluation of costs associated with tolvaptan-mediated length-ofstay reduction among heart failure patients with hyponatremia in the US, based on the EVEREST trial," Journal of Medical Economics, vol. 15, no. 2, pp. 276-284, 2012.

[24] A. K. Salahudeen, N. Ali, M. George, A. Lahoti, and S. Palla, "Tolvaptan in hospitalized cancer patients with hyponatremia: A double-blind, randomized, placebo-controlled clinical trial on efficacy and safety," Cancer, vol. 120, no. 5, pp. 744-751, 2014. 


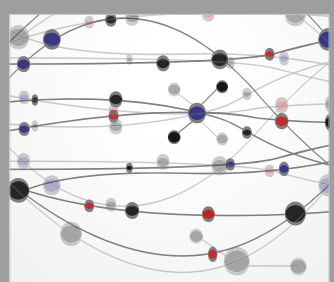

The Scientific World Journal
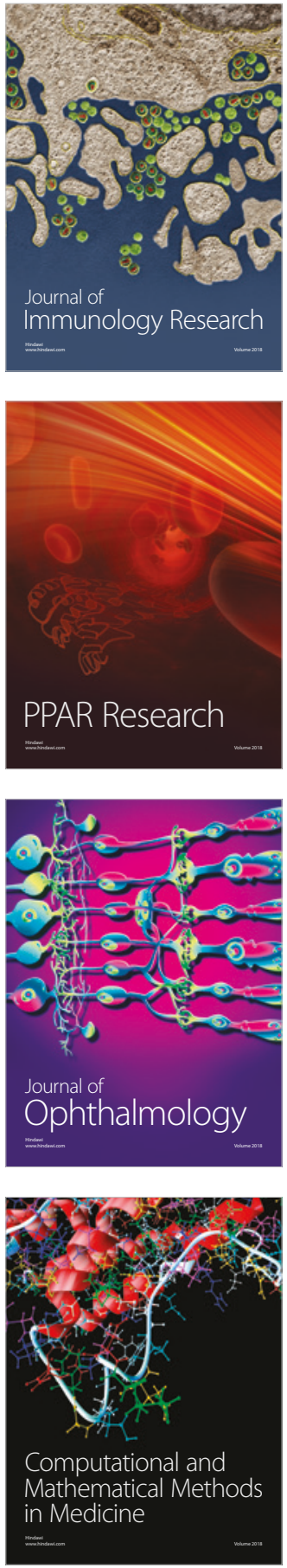

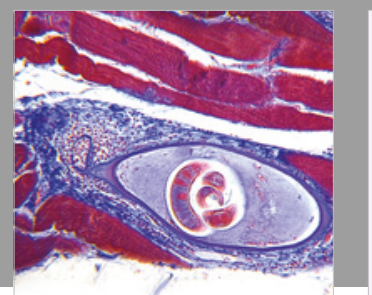

Gastroenterology Research and Practice

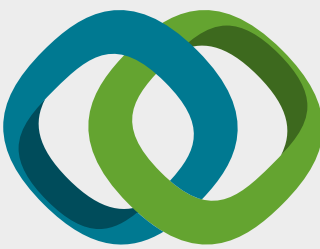

\section{Hindawi}

Submit your manuscripts at

www.hindawi.com
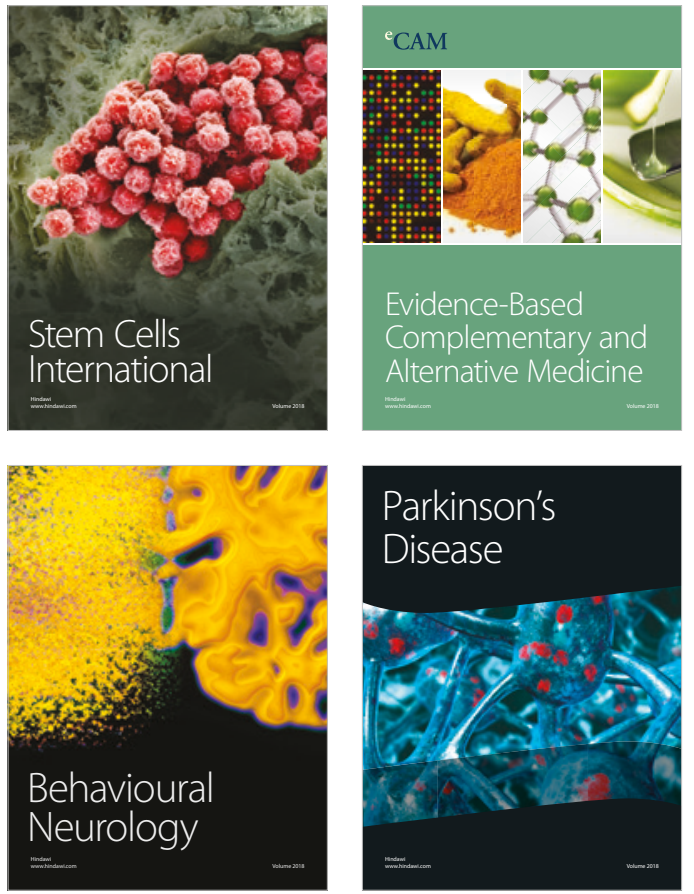

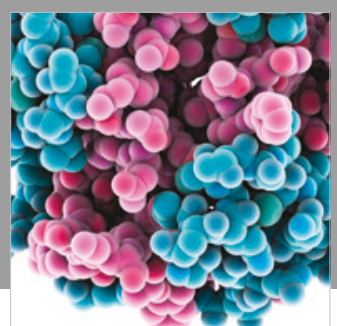

ournal of

Diabetes Research

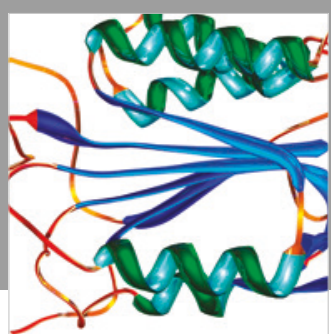

Disease Markers
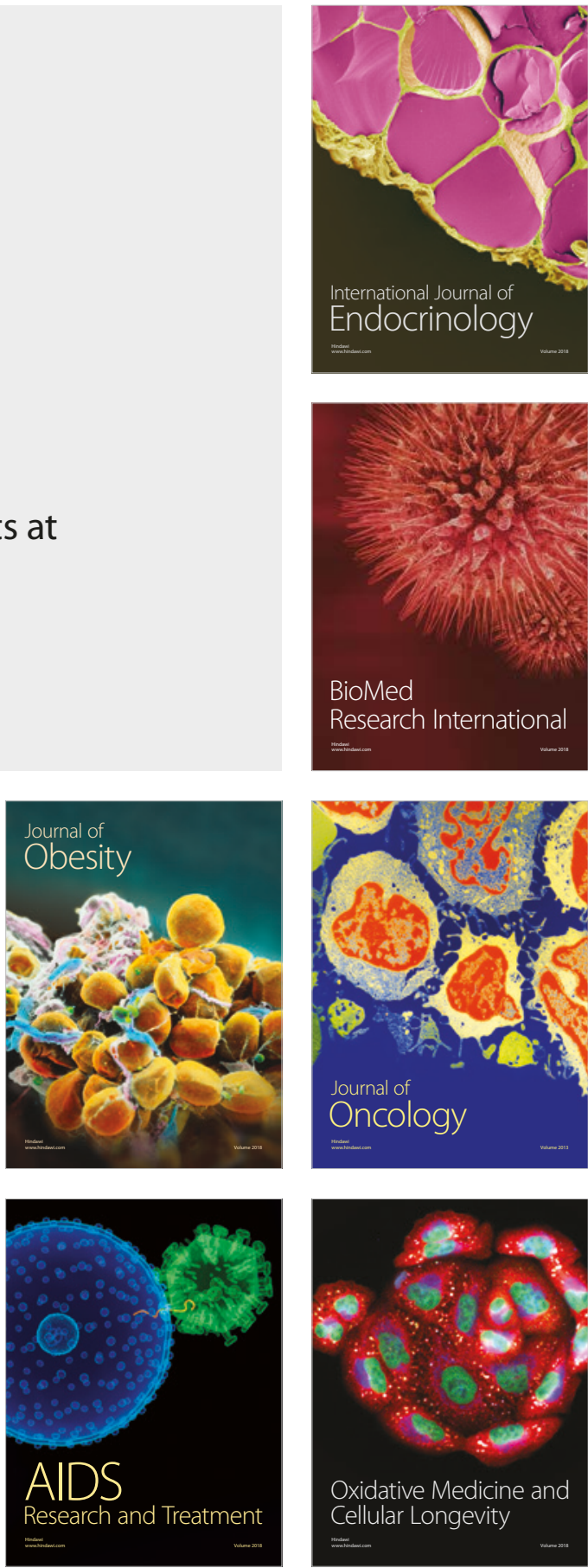